\title{
Inflaton Condensate Fragmentation in Hybrid Inflation Models
}

\author{
JOHN MCDONALD ${ }^{1}$ \\ Theoretical Physics Division, University of Liverpool, Liverpool L69 3BX, England
}

October 24, 2018

\begin{abstract}
Inflation ends with the formation of a Bose condensate of inflatons. We show that in hybrid inflation models this condensate is typically unstable with respect to spatial perturbations and can fragment to condensate lumps. The case of Dterm inflation is considered as an example and it is shown that fragmentation occurs if $\lambda \gtrsim 0.2 g$, where $\lambda$ is the superpotential coupling and $g$ is the $U(1)_{F I}$ gauge coupling. Condensate fragmentation can result in an effective enhancement of inflaton annihilations over decays as the main mode of reheating. In the case of D-term inflation models in which the Standard Model fields carry $U(1)_{F I}$ charges, if condensate fragmentation occurs then reheating is dominated by inflaton annihilations, typically resulting in the overproduction of thermal gravitinos. Fragmentation may also have important consequences for SUSY flat direction dynamics and for preheating.
\end{abstract}

${ }_{1}^{1 \text { mcdonald@sune.amtp.liv.ac.uk }}$ 


\section{Introduction}

A common feature of the cosmology of particle physics models is the formation of Bose condensates of scalar particles. Examples include axion condensates [1], condensates of squarks and sleptons along flat directions of the minimal supersymmetric (SUSY) standard model (MSSM) (Affleck-Dine condensates [2, 3, 田) and inflaton condensates which form at the end of inflation and whose decay is responsible for reheating the Universe [5]. It is usually assumed that the scalar particles in the condensate are noninteracting, corresponding to coherent oscillations in a purely $\phi^{2}$ potential. However, in many cases this is not true. In the case of axions, deviation of the angular pseudoNambu Goldstone axion potential from a pure $\phi^{2}$ potential implies an attractive force between the axions which results in the growth of spatial perturbations and the formation of axion miniclusters [6]. In the case of the Affleck-Dine condensate, deviation from the $\phi^{2}$ potential, either due to the flattening of the potential above the messenger field mass (gauge-mediated SUSY breaking [7, 8) or due to radiative corrections from gaugino loops (gravity-mediated SUSY breaking [9, 10]) results the fragmentation of the condensate to form Q-balls [8, 9, 10, 11]. Thus the conventional view of cosmological condensates as being spatially homogeneous coherently oscillating scalar fields is not generally true. In particular, when the potential is 'flatter-than- $\phi^{2}$, meaning $\operatorname{Min}\left(V(\phi) / \phi^{2}\right)$ is at $\phi \neq 0$ (with $V(0)=0$ ), the condensate is unstable with respect to spatial perturbations and fragments to non-topological solitons which we will refer to as condensate lumps.

Here we consider the question of the stability of the inflaton condensate with respect to spatial perturbations and the consequences of its fragmentation. The most natural inflation models are hybrid inflation models [12], which, unlike the case of single-field inflation models, allow inflation to occur without requiring couplings to be very small. We will therefore focus on hybrid inflation models in the following.

Although our results for inflaton condensate fragmentation can apply to hybrid inflation models in general, we will focus on the case of SUSY hybrid inflation models [13], 14, 15], which will allow us to illustrate the general phenomenon of inflaton con- 
densate fragmentation whilst applying the results to a case of considerable interest. SUSY hybrid inflation models are either of the F-term [13] or D-term [14, [16] type. The most interesting are the D-term models, which can evade the so-called $\eta$-problem i.e. the flatness of the inflaton potential in the presence of supergravity corrections [15]. We will therefore focus on D-term inflation, whilst presenting the results in a form that will allow them to be applied to other hybrid inflation models.

Recently it has been shown that it is also possible for inflation to end via "tachyonic preheating" i.e. the rapid growth of spatial perturbations of the inflaton field in the presence of a tachyonic potential [17, 18, 19]. The mode by which hybrid inflation ends (inflaton condensate fragmentation or tachyonic preheating) will be sensitive to the initial conditions at the phase transition ending hybrid inflation, in particular the rate of roll of the homogeneous scalar field relative to the rate of growth of the spatial perturbations. This requires a full analysis of the dynamics of the inflaton field, including the effect of radiative corrections to the inflaton potential [20]. Since in this paper we wish to study the growth of spatial perturbations of a homogeneous hybrid inflation condensate in general, using D-term inflation as a particular example, we will assume throughout that a coherently oscillating scalar field condensate initially exists.

The paper is organised as follows. In Section 2 we review the D-term hybrid inflation model. In Section 3 we discuss condensate instability in hybrid inflation models. In Section 4 we consider the evolution of spatial perturbations of a coherently oscillating condensate. In Section 5 we apply the results to the case of D-term inflation. In Section 6 we consider possible consequences of inflaton condensate fragmentation, in particular the enhancement of annihilations as a mode of reheating. In Section 7 we comment on the relationship between tachyonic preheating and inflaton condensate fragmentation. In Section 8 we present our conclusions.

\section{D-term Hybrid Inflation}

The superpotential of D-term inflation models is [14

$$
W=\lambda S \Phi_{+} \Phi_{-},
$$


resulting in a scalar potential

$$
V=\lambda^{2}|S|^{2}\left(\left|\Phi_{+}\right|^{2}+\left|\Phi_{-}\right|^{2}\right)+\lambda^{2}\left|\Phi_{+}\right|^{2}\left|\Phi_{-}\right|^{2}+\frac{g^{2}}{2}\left(\left|\Phi_{+}\right|^{2}-\left|\Phi_{-}\right|^{2}+\xi\right)^{2},
$$

where $\operatorname{Re}(S)$ is the gauge singlet inflaton, $\Phi_{ \pm}$are fields with charges \pm 1 with respect to a Fayet-Illiopoulos $U(1)$ gauge symmetry, $U(1)_{F I}$, and $\xi>0$ is the Fayet-Illiopoulos term. For $|S|>\left|S_{c}\right|=g \sqrt{\xi} / \lambda$, the minimum of $V\left(\Phi_{+}, \Phi_{-} ;|S|\right)$ is at $\Phi_{ \pm}=0$. With $\Phi_{ \pm}=0$, the tree-level $S$ potential is flat with $V=V_{o} \equiv g^{2} \xi^{2} / 2\left(\xi^{1 / 2} \approx 8.5 \times 10^{15} \mathrm{GeV}\right.$ from COBE normalization [15]). One-loop corrections result in a potential for $S$ which causes $S$ to slow-roll towards $S=0[14]$. Once $|S|<\left|S_{c}\right|$, the minimum of the potential for a given value of $|S|$ is at $\Phi_{+}=0$ and

$$
\left|\Phi_{-}\right|=\sqrt{\xi-\frac{\lambda^{2}|S|^{2}}{g^{2}}}
$$

(In the following we may consider $S$ and $\Phi_{-}$to be real.) Thus the expectation value of

$\Phi_{-}$at the minimum of its potential is a function of the value of $S$. The mass squared terms along the $S, \Phi_{+}$and $\Phi_{-}$directions as a function of $|S|$ and the $\Phi_{-}$expectation value Eq. (3) are $m_{S}^{2}=\lambda^{2}\left|\Phi_{-}\right|^{2}, m_{\Phi_{+}}^{2}=\lambda^{2}\left|\Phi_{-}\right|^{2}+2 \lambda^{2}|S|^{2}$ and $m_{\Phi_{-}^{\prime}}^{2}=m_{A}^{2}=2 g^{2}\left|\Phi_{-}\right|^{2}$, where $m_{\Phi_{-}^{\prime}}$ is the mass at $\Phi_{-} \neq 0$ minimum Eq. (3) and $A$ is the $U(1)_{F I}$ gauge boson.

\section{Condensate Instability in Hybrid Inflation Mod- els}

The dependence of the minimum of the $\Phi_{-}$potential on the value of the $S$ field is the reason for the instability of the inflaton condensate. Once $|S|<\left|S_{c}\right|, S$ and $\Phi_{-}$ oscillate about the minimum of their potentials. Oscillations begin once $m_{S}>H$. In the case of D-term inflation, this is satisfied once $|S|^{2} /\left|S_{c}\right|^{2}=1-4 \pi g^{2} \xi / 3 \lambda^{2} M_{P l}^{2}=$ $1-2 \times 10^{-6} g^{2} / \lambda^{2}$ (using $m_{S}=\lambda\left|\Phi_{-}\right|$, with $\left|\Phi_{-}\right|$as given by Eq. (3)), so $S$ oscillations typically begin when $|S|$ is close to $\left|S_{c}\right|$. The equation on motion for the inflaton, in terms of the conventionally normalized real scalar field $s=\sqrt{2} \operatorname{Re}(S)$, is

$$
\ddot{s}+3 H \dot{s}-\frac{\underline{\nabla}^{2}}{a^{2}} s=-\lambda^{2} s\left|\Phi_{-}\right|^{2},
$$


where $H=\dot{a} / a$ is the expansion rate and $a$ is the scale factor. Suppose we consider the growth of a small spatial perturbation of $s$. As $s$ decreases below $s_{c}$, the mean value of the oscillating $\Phi_{-}$field at a point in space will be approximately equal to the value at minimum of the $\Phi_{-}$potential at that point in space, which depends of $s(\mathbf{x}, t)$. So if we average over the coherent oscillations of $\Phi_{-}$about the minimum and replace $\Phi_{-}$ by the value $\Phi_{-}(s)$ at the minimum of its potential, the $s$ equation of motion becomes

$$
\ddot{s}+3 H \dot{s}-\frac{\underline{\nabla}^{2}}{a^{2}} s \approx-\lambda^{2} \xi s+\frac{\lambda^{4} s^{3}}{2 g^{2}} .
$$

Therefore the $s$ scalar field and perturbations will evolve as if the $s$ field had a potential

$$
V_{e f f}(s) \approx \frac{\lambda^{2} \xi s^{2}}{2}-\frac{\lambda^{4} s^{4}}{8 g^{2}}
$$

This is a flatter than $s^{2}$ potential, corresponding to an attractive interaction amongst the $s$ scalars and a negative pressure in the condensate [21, 22]. Therefore spatial perturbations of the $s$ condensate will grow, eventually becoming non-linear and resulting in fragmentation into condensate lumps [8, 9, 10]. The procedure of averaging over coherent oscillations of the $\Phi_{-}$field is well-defined if $m_{\Phi_{-}^{\prime}}$ is large compared with $m_{S}$, which is true if $\sqrt{2} g$ is large compared with $\lambda$, and we will focus on this case. In the case where one cannot first average over the $\Phi_{-}$oscillations the combined dynamics of the $S$ and $\Phi_{-}$field will be more complicated. A particular case of this is F-term hybrid inflation, for which there is only a single coupling in the scalar potential such that the condition $\lambda=\sqrt{2} g$ is effectively satisfied [13, 15]. In this case there exists an exact solution of the scalar field equations such that the inflaton is described by an effective potential of the form $a s^{2}-b|s|^{3}+c s^{4}(a, b, c>0)$ [24.

Although we have derived $V_{e f f}(s)$ for the example of D-term inflation, we emphasize that a $-s^{4}$ attractive interaction is a generic feature of all hybrid inflation models for which we can average over the oscillations of the field terminating inflation prior to discussing the dynamics of the inflaton. Therefore our analysis may be readily applied to other hybrid inflation models. 


\section{Evolution of Perturbations}

We next consider the growth of spatial perturbations and the fragmentation of the inflaton condensate. The linear growth of perturbations has been discussed for a complex scalar field in the context of Q-ball formation in [8], using the approach of [25]. Here we adapt this approach to the case of a real scalar field in the expanding Universe. The equation of motion for a real scalar field $\Phi$ is

$$
\ddot{\Phi}+3 H \dot{\Phi}-\frac{\underline{\nabla}^{2}}{a^{2}} \Phi=-\frac{\partial V(\Phi)}{\partial \Phi} .
$$

We will assume throughout that $V(\Phi)$ is a polynomial with $V(\Phi)=V(-\Phi)$. We define $\Phi=\left(a_{o} / a\right)^{3 / 2} \phi$, where $a_{o}$ is the scale factor when the coherent oscillations begin. The equation of motion then becomes

$$
\ddot{\phi}-\frac{\underline{\nabla}^{2}}{a^{2}} \phi=-\frac{\partial U(\phi)}{\partial \phi},
$$

where

$$
\frac{\partial U(\phi)}{\partial \phi}=\left(\frac{a}{a_{o}}\right)^{3 / 2} \frac{\partial V(\Phi)}{\partial \Phi}+\Delta_{H} \phi
$$

where

$$
\Delta_{H}=-\frac{3}{2}\left(\dot{H}+\frac{3}{2} H^{2}\right) .
$$

With $\phi=R \operatorname{Sin} \Omega$, the equation of motion becomes

$$
\left(\ddot{R}-R \dot{\Omega}^{2}-\frac{\nabla^{2}}{a^{2}} R+\frac{R\left(\partial_{i} \Omega\right)^{2}}{a^{2}}\right) \operatorname{Sin} \Omega+\left(-R \ddot{\Omega}-2 \dot{R} \dot{\Omega}+\frac{2 \partial_{i} R \partial_{i} \Omega}{a^{2}}+R \frac{\frac{\nabla}{2}^{2}}{a^{2}} \Omega\right) \operatorname{Cos} \Omega=-\frac{\partial U(\phi)}{\partial \phi},
$$

where $\partial_{i}=\partial / \partial x_{i}(i=1,2,3)$. Multiplying the equation by $\operatorname{Sin} \Omega$ and averaging over coherent oscillations gives

$$
\ddot{R}-R \dot{\Omega}^{2}-\frac{\underline{\nabla}^{2}}{a^{2}} R+\frac{R\left(\partial_{i} \Omega\right)^{2}}{a^{2}}=-\frac{\partial U_{e f f}(R)}{\partial R},
$$

where

$$
\frac{\partial U_{e f f}(R)}{\partial R}=\frac{2}{R}\left\langle\phi \frac{\partial U}{\partial \phi}\right\rangle
$$


and where $<f(\Omega)>\equiv \frac{1}{2 \pi} \int_{0}^{2 \pi} f(\Omega) d \Omega$ denotes averaging over oscillations. $\left(\Delta_{H}\right.$ is effectively constant on the timescale of coherent oscillations. In fact, since $\Delta_{H}$ is at most of the order of $H^{2}$, the $\Delta_{H}$ term in general contributes a negligible correction to the $\phi$ mass squared term. Thus we take $\Delta_{H}=0$ in the following.) Multiplying both sides by $\operatorname{Cos} \Omega$ and averaging gives

$$
\ddot{\Omega}+2 \frac{\dot{R}}{R} \dot{\Omega}-\frac{2}{R} \frac{\partial_{i} R \partial_{i} \Omega}{a^{2}}-\frac{\nabla^{2}}{a^{2}} \Omega=0 .
$$

In this we are assuming that $R$ and $\dot{\Omega}$ do not vary much over the period of the oscillations. In practice we will be applying this method to the case of a $-\Phi^{4}$ interaction term in the potential. Therefore this method is accurate if the $-\Phi^{4}$ term is a small perturbation of the $\Phi^{2}$ term. (In the pure $\Phi^{2}$ limit $R$ and $\dot{\Omega}$ are constant.)

With $R=R+\delta R(\mathbf{x}, t)$ and $\Omega=\Omega(t)+\delta \Omega(\mathbf{x}, t)$, the perturbation equations are

$$
\delta \ddot{R}-\dot{\Omega}^{2} \delta R-2 \dot{\Omega} \delta \dot{\Omega} R-\frac{\underline{\nabla}^{2}}{a^{2}} \delta R=-\left(\frac{\partial^{2} U_{e f f}}{\partial R^{2}}\right)_{R(t)} \delta R
$$

and

$$
\delta \ddot{\Omega}+2 \frac{\dot{R}}{R} \delta \dot{\Omega}+2 \frac{\dot{\Omega}}{R} \delta \dot{R}-2 \frac{\dot{R} \dot{\Omega}}{R^{2}} \delta R-\frac{\underline{\nabla}^{2}}{a^{2}} \delta \Omega=0 .
$$

Assuming the perturbations have the form $\delta R=\delta R_{o} e^{S(t)-i \mathbf{k} \cdot \mathbf{x}}, \delta \Omega=\delta \Omega_{o} e^{S(t)-i \mathbf{k} \cdot \mathbf{x}}$ [8, 25], the perturbation equations become

$$
\left(\dot{\alpha}+\alpha^{2}-\dot{\Omega}^{2}+\frac{\mathbf{k}^{2}}{a^{2}}+U_{e f f}^{\prime \prime}\right) \delta R=2 \alpha R \dot{\Omega} \delta \Omega
$$

and

$$
\left(\dot{\alpha}+\alpha^{2}+\frac{\mathbf{k}^{2}}{a^{2}}+2 \frac{\dot{R}}{R} \alpha\right) \delta \Omega=\delta R\left(\frac{2 \dot{R} \dot{\Omega}}{R^{2}}-\frac{2 \dot{\Omega} \alpha}{R}\right),
$$

where $\alpha=\dot{S}$. Combining these gives a dispersion relation 25, 8],

$$
\left(\dot{\alpha}+\alpha^{2}-\dot{\Omega}^{2}+\frac{\mathbf{k}^{2}}{a^{2}}+U_{e f f}^{\prime \prime}\right)\left(\dot{\alpha}+\alpha^{2}+\frac{\mathbf{k}^{2}}{a^{2}}+2 \frac{\dot{R}}{R} \alpha\right)=2 \alpha R \dot{\Omega}\left(\frac{2 \dot{R} \dot{\Omega}}{R^{2}}-\frac{2 \dot{\Omega} \alpha}{R}\right) \text {. }
$$

In the case where there is no expansion the amplitude of oscillation is constant and so we have $\dot{R}=0$. A growing perturbation solution is then given by [25], 8]

$$
\alpha^{2}=\frac{\frac{\mathbf{k}^{2}}{a^{2}}\left(\frac{U_{e f f}^{\prime}}{R}-U_{e f f}^{\prime \prime}\right)}{\left(\frac{3 U_{e f f}^{\prime}}{R}+U_{e f f}^{\prime \prime}\right)}
$$


and $\dot{\alpha}=0$. In deriving this we have used $\dot{\Omega}^{2}=U_{\text {eff }}^{\prime} / R$ (from Eq. (12) with constant $R$ ). This solution exists if $\mathbf{k}^{2} / a^{2}$ is less than $\mathbf{k}_{\text {max }}^{2} / a^{2}=\left(\frac{U_{e f f}^{\prime}}{R}-U_{\text {eff }}^{\prime \prime}\right)$. (In obtaining Eq. (20) it is assumed that $16\left(\mathbf{k}^{2} / a^{2}\right) U_{\text {eff }}^{\prime} / R$ is small compared with $\left(3 U_{\text {eff }}^{\prime} / R+U_{\text {eff }}^{\prime \prime}\right)^{2}$, which is satisfied for all $\mathbf{k}$ up to $\mathbf{k}_{\max }$ in the case where the $-\Phi^{4}$ potential term is small compared with the $\Phi^{2}$ term.)

In the case with expansion we generally have to solve the equations of motion and perturbation equations numerically. However, for condensate fragmentation we will be mostly interested in the case where $\mathbf{k}^{2}=\mathbf{k}_{\text {max }}^{2}$, corresponding to the largest value of $\alpha$ at a given time and so the first perturbation mode to go non-linear. We will also be considering oscillation amplitudes such that the $-\Phi^{4}$ potential term is small compared with the $\Phi^{2}$ term. In this case $R$ may be considered constant throughout. Then if $\dot{\alpha}$ is non-zero, the solution Eq. (19) generalizes to

$$
\tilde{\alpha}^{2}=\frac{\frac{\mathbf{k}^{2}}{a^{2}}\left(\frac{U_{e f f}^{\prime}}{R}-U_{e f f}^{\prime \prime}\right)}{\left((4 \gamma-1) \frac{U_{e f f}^{\prime}}{R}+U_{e f f}^{\prime \prime}\right)}
$$

where $\tilde{\alpha}=\alpha^{2}+\dot{\alpha}$ and $\gamma=\alpha^{2} / \tilde{\alpha}^{2}$. Since typically $|\dot{\alpha} / \alpha| \approx H$, we see that Eq. (20) will be approximately correct for the case $\mathbf{k}^{2}=\mathbf{k}_{\max }^{2}$ so long as $\alpha\left(\mathbf{k}_{\max }\right)>\left|\frac{\dot{\alpha}}{\alpha}\right| \approx H$. The solution of the perturbation equations is then

$$
\delta R \approx \delta R_{o} \exp \left(\int \alpha d t\right) e^{i \mathbf{k} \cdot \mathbf{x}}
$$

and

$$
\delta \Omega \approx \delta \Omega_{o} \exp \left(\int \alpha d t\right) e^{i \mathbf{k} \cdot \mathbf{x}}
$$

We next apply the above to the case of the generic attractive $-\Phi^{4}$ interaction of hybrid inflation models,

$$
V(\Phi)=\frac{m^{2} \Phi^{2}}{2}-\frac{\eta \Phi^{4}}{4}
$$

From Eq. (13),

$$
\frac{\partial U_{e f f}(R)}{\partial R}=\frac{2}{R}\left\langle m^{2} \phi^{2}-\eta \phi^{4}\left(\frac{a_{o}}{a}\right)^{3}\right\rangle=\frac{2}{R}\left(\frac{m^{2} R^{2}}{2}-\frac{3 \eta R^{4}}{8}\left(\frac{a_{o}}{a}\right)^{3}\right)
$$


where we have used $<\operatorname{Sin}^{2} \Omega>=1 / 2$ and $<\operatorname{Sin}^{4} \Omega>=3 / 8$. Thus

$$
U_{\text {eff }}(R)=\frac{m^{2} R^{2}}{2}-\frac{3 \eta R^{4}}{16}\left(\frac{a_{o}}{a}\right)^{3} .
$$

Thus from Eq. (20) we find

$$
\alpha=\left(\frac{a_{o}}{a}\right)^{3 / 2}\left(\frac{3 \eta R^{2}}{8 m^{2}}\right)^{1 / 2}\left(1-\frac{9 \eta R^{2}}{8 m^{2}}\left(\frac{a_{o}}{a}\right)^{3}\right)^{-1 / 2} \frac{|\mathbf{k}|}{a} .
$$

Eq. (27) is strictly valid only if the $-\Phi^{4}$ term is a small perturbation of the $\Phi^{2}$ term. In this case $R$ will be essentially constant (equal to its initial value $R_{o}$ ) and $\left(1-\frac{9 \eta R^{2}}{8 m^{2}}\left(\frac{a_{o}}{a}\right)^{3}\right)$ will be approximately equal to 1 , conditions which we will assume to be satisfied in the following.

The growth of the perturbations is then given by,

$$
\frac{\delta R}{R} \approx \frac{\delta R_{o}}{R_{o}} \exp \left[\left(\frac{3 \eta R_{o}^{2}}{8 m^{2}}\right)^{1 / 2} \frac{|\mathbf{k}|}{a H_{o}}\left(\frac{2}{5-2 n}\right)\left(\frac{a_{o}}{a}\right)^{3 / 2-n}\left(\left(\frac{a}{a_{o}}\right)^{5 / 2-n}-1\right)\right] .
$$

In this we have used $H=H_{o}\left(a_{o} / a\right)^{n}$, where $n$ will be between 0 and $3 / 2$ as the $\Phi$ oscillations develop from the end of inflation to an approximately $\Phi^{2}$ potential. The condition for fragmentation to occur is that $\delta R / R \gtrsim 1$. The largest growth at a given time corresponds to the mode $\mathbf{k}_{\max }$, where

$$
\frac{\mathbf{k}_{\max }^{2}}{a^{2}}=\left(\frac{U_{\text {eff }}^{\prime}}{R}-U_{\text {eff }}^{\prime \prime}\right)=\frac{3}{2} \eta R^{2}\left(\frac{a_{o}}{a}\right)^{3} .
$$

This determines the radius of the condensate lumps when the condensate fragments, $r_{l}$,

$$
r_{l} \approx \frac{\pi a}{\left|\mathbf{k}_{\max }\right|}=\left(\frac{2}{3}\right)^{1 / 2}\left(\frac{a}{a_{o}}\right)^{3 / 2} \frac{\pi}{\left(\eta R^{2}\right)^{1 / 2}} .
$$

This is really the initial radius of the lumps immediately after fragmentation, and the lump will subsequently relax to its stable configuration, in which the attractive potential term is balanced by the gradient term in the equation of motion. However, the radius of the stable configuration is similar to that of the initial lump, since for a stable configuration of the form $\phi(r, t) \approx \phi(r) \operatorname{Sin}(m t)$, the equation for $\phi(r)$ is $\partial^{2} \phi(r) / \partial r^{2}+(2 / r) \partial \phi(r) \partial r \approx \operatorname{Sin}^{-1}(m t) \partial \delta V / \partial \phi$, where $V=m^{2} \phi^{2} / 2+\delta V$. For a stable 
lump of radius $r_{s}$ and field amplitude $\phi$ we therefore expect that $r_{s}^{2} \approx \phi|\partial \delta V / \partial \phi|^{-1}$ (the left hand side of the $\phi(r)$ stable lump equation being $\sim \phi / r^{2}$ ). For $\delta V=-\eta \phi^{4} / 4$ and $\phi \approx R$ this implies that $r_{s} \approx\left(\eta R^{2}\right)^{-1 / 2}$.

To give a condition for inflaton condensate fragmentation, we use $\mathbf{k}=\mathbf{k}_{\max }$. The condition for fragmentation to occur is then

$$
\frac{1}{2 m}\left(\frac{3 \eta R_{o}^{2}}{2}\right) \frac{1}{H_{o}}\left(\frac{2}{5-2 n}\right)\left(\left(\frac{a_{o}}{a}\right)^{1 / 2}-\left(\frac{a_{o}}{a}\right)^{3-n}\right)>\beta \equiv \log \left(\frac{R_{o}}{\delta R_{o}}\right) .
$$

As $a$ increases, the left hand side of Eq. (31) is maximized for $\frac{a_{o}}{a}=\left(\frac{1}{2(3-n)}\right)^{\frac{2}{5-2 n}}$ and then decreases. Condensate fragmentation in hybrid inflation models must therefore occur soon after coherent oscillations begin if it is to occur at all.

Assuming that the $-\Phi^{4}$ contribution to the potential is small, the coherent oscillations are approximately $\Phi^{2}$ and so $n=3 / 2$, such that maximum growth occurs at $\frac{a_{o}}{a}=\frac{1}{3}$. The condition for condensate fragmentation is then

$$
\frac{1}{2 \sqrt{3}} \frac{\eta R_{o}^{2}}{m H_{o}} \gtrsim \beta
$$

where $H_{o}$ is calculated using for the energy density $\rho=m^{2} R_{o}^{2} / 2$.

\section{Application to D-term Inflation}

For the case of D-term inflation, $m^{2}=\lambda^{2} \xi$ and $\eta=\lambda^{4} / 2 g^{2}$. In order that the $-\Phi^{4}$ contribution to the potential is small, we choose $R_{o}=s_{c} / 2$, such that $R_{o}^{2}=$ $g^{2} \xi / 2 \lambda^{2}$. (The perturbations will start to grow as soon as coherent oscillations begin with amplitude $s \approx s_{c}$. However, as we cannot apply our method to calculate the growth for oscillation amplitudes between $s_{c}$ and $s_{c} / 2$, we are in fact underestimating the total growth.) Thus from Eq. (32) the condition for condensate fragmentation becomes

$$
\frac{\lambda}{g} \frac{1}{\beta} \gtrsim \frac{8 \sqrt{2 \pi} \xi^{1 / 2}}{M_{P l}} \approx 0.014
$$

In order to complete the fragmentation condition we need the value of $\beta$. The seed perturbations of the inflaton field are expected to come from quantum de Sitter 
fluctuations of the scalar field during inflation. Modes with wavenumber $k$ large compared w ith $H$ will be excited by the increase of the inflaton mass at the end of inflation from approximately zero to $m_{S} \approx \lambda \xi^{1 / 2}$. The largest wavenumber excited typically corresponds to $k_{m} \approx m_{S}$, with amplitude $\delta s \approx m_{S} /(2 \pi)$ [23]. Since $\left|\mathbf{k}_{\max }\right| / a \approx \sqrt{3 / 2} \lambda \xi^{1 / 2} \approx k_{m}$ at $a \approx a_{o}$, seed perturbations of wavenumber $\mathbf{k}_{\max } / a$ will exist after inflation ends, with

$$
\beta \approx \log \left(\frac{2 \pi s_{c}}{m_{S}}\right)=\log \left(\frac{2 \sqrt{2} \pi g}{\lambda^{2}}\right),
$$

where we have used $\delta R_{o} / R_{o} \approx \delta s / s_{c}$. So with $\lambda, g \gtrsim 0.1, \beta \approx 5-10$ is a typical value. Therefore, using $\beta=10$, we find that the condition for fragmentation to occur is

$$
\lambda \gtrsim 0.2 g
$$

Since this neglects the growth of perturbations for oscillation amplitudes between $s_{c}$ and $s_{c} / 2$, the true lower bound on $\lambda$ for fragmentation is likely to be smaller. The radius of the condensate lump relative to the horizon radius when the condensate fragments is then

$$
\frac{r_{l}}{H^{-1}} \approx\left(\frac{8 \pi^{3}}{9}\right)^{1 / 2} \frac{g}{\lambda} \frac{\xi^{1 / 2}}{M_{P l}}=3.7 \times 10^{-3} \frac{g}{\lambda} .
$$

The condition for the approximations leading to Eq. (22) to be consistent, $\alpha\left(\mathbf{k}_{\max }\right)>$ $\left|\frac{\dot{\alpha}}{\alpha}\right|=5 H / 2$ at $\frac{a_{o}}{a}=\frac{1}{3}$ (where we assume $H \propto a^{-3 / 2}$ for $s<s_{c} / 2$ and we have used $\alpha \propto a^{-5 / 2}$ from Eq. (27)), is satisfied if

$$
\frac{\lambda}{g}>\frac{40 \sqrt{2 \pi} \xi^{1 / 2}}{M_{P l}} \approx 0.07
$$

So if the fragmentation condition Eq. (35) is satisfied then the approximations are consistent.

Therefore in D-term inflation models condensate fragmentation is likely to occur if $\lambda \gtrsim 0.2 g$. Since the true lower bound on $\lambda$ for fragmentation is likely to be smaller, it is probable that condensate fragmentation will occur if $\lambda$ and $g$ both take values in the natural range 0.1-1. 


\section{Enhancement of Inflaton Annihilations By Con- densate Fragmentation}

We next consider the possible consequences of inflaton condenate fragmentation. One potentially important consequence is that inflaton annihilations are effectively enhanced compared with the case of a homogeneous condensate and may dominate over decays as the primary mode of reheating.

We first show that once the condensate fragments the particles within the condensate lump decouple from the expansion of the Universe, such that the number density and field amplitude inside the condensate lumps remains constant. This will be true if the force on the particles due to scalar interactions is greater than the gravitational force responsible for slowing the expansion of the Universe. Suppose the energy density is dominated by a pressureless homogeneous energy density $\bar{\rho}$. (For the case of inflaton condensate fragmentation this is not strictly true, since $\delta \rho / \bar{\rho} \approx 1$ when the condensate fragments. However, it is useful to make this assumption in order to obtain an expression that can be applied to scalar field models in general.) Suppose then we consider a spherical lump of radius $r$. The gravitational acceleration acting on a particle at the surface of the lump is then

$$
\ddot{r}=-\frac{4 \pi \bar{\rho}}{3 M_{P l}^{2}} r
$$

If the force due to the attractive scalar interaction produces a smaller acceleration than this, the particles will follow the expansion of the Universe, otherwise they will decouple from expansion. To estimate the force due to the scalar interaction consider a sphere of radius $r$ and with a fixed number of scalar particles $N$,

$$
N=\frac{4 \pi r^{3}}{3}\left(\frac{m \phi^{2}}{2}\right),
$$

where the number density of scalars is $n=m \phi^{2} / 2$ and where for simplicity we have considered a constant amplitude $\phi$ for the coherently oscillating field inside the sphere.

This gives $\phi$ as a function of $r$. With $V(\phi)=\frac{m^{2} \phi^{2}}{2}+\delta V$, the total potential energy of 
the sphere is

$$
E_{p o t}=\frac{4 \pi r^{3}}{3}\left(\frac{m^{2} \phi^{2}}{2}+\delta V\right)=m N+\frac{4 \pi r^{3}}{3} \delta V .
$$

Thus the force due to the scalars is

$$
F_{s}=-\frac{d E_{p o t}}{d r}=-\left(4 \pi r^{2} \delta V-\frac{6 N}{m r} \frac{\partial \delta V}{\partial \phi^{2}}\right) .
$$

For the case $\delta V=-\eta \phi^{4} / 4$, the force is $F_{s}=-\pi \eta \phi^{4} r^{2}$. The condition that the acceleration due to scalar attraction is larger than the gravitational acceleration is then

$$
\phi^{4}>\frac{m H^{2}}{2 \pi \eta r} .
$$

Since condensate fragmentation occurs soon after coherent oscillations begin (at $\partial V / \partial \phi \approx$ 0 ), we have $\phi^{2} \approx m^{2} / \eta$. With $r \approx r_{l}$ (with $a \approx a_{o}$ in Eq. (30)) Eq. (42) becomes

$$
\frac{m^{3}}{\eta^{3 / 2} R}>\left(\frac{3}{2}\right)^{1 / 2} \frac{H^{2}}{2 \pi^{2}} \text {. }
$$

Note that if this condition is satisfied then from Eq. (38) we have $|\ddot{r} / r| \gtrsim H^{2}$, which can be rewritten as $|\ddot{r}| H^{-1} \gtrsim H r \equiv \dot{r}$. Therefore the attractive force between the scalars will bring the expansion of the lump to a halt within an expansion time, $\delta t \approx H^{-1}$. For the case of D-term inflation, Eq. (43) is satisfied if

$$
\lambda<\left(\frac{\sqrt{6} \pi M_{P l}^{2}}{\xi}\right)^{1 / 2} \approx 4 \times 10^{3},
$$

which is generally strongly satisfied. Thus the scalars in the condensate lumps decouple from the gravitational expansion.

To see how condensate fragmentation effectively enhances annihilations, consider an interaction $\lambda^{2}|S|^{2}\left|Q_{i}\right|^{2}$ between inflatons and light scalars $Q_{i}$ (in D-term inflation $Q_{i}$ will correspond to MSSM scalars [16]). We will calculate the annihilation rate assuming no Bose enhancement (i.e. no parametric resonant decay of the condensate), in which case the annihilation rate simply corresponds to the perturbative annihilation rate of the scalars in the condensate. The average of the annihilation cross-section times relative particle velocity in the $v \rightarrow 0$ limit is [26]

$$
<\sigma v>_{a n n}=\frac{\lambda^{4}}{64 \pi m_{S}^{2}} \text {. }
$$


With the $s$ number density in the condensate given by $n=m_{S} s^{2} / 2$, the annihilation rate of scalars in the condensate is then

$$
\Gamma_{a n n}=n<\sigma v>_{a n n}=\frac{\lambda^{4} s^{2}}{128 \pi m_{s}} .
$$

The condition for scalars to annihilate is then $\Gamma_{a n n}>H \equiv H_{o}\left(a_{o} / a\right)^{3 / 2}$. We can now see why annihilation is effectively enhanced when the condensate fragments. In the case of a homogeneous condensate, the scalars are freely expanding and $s^{2} \propto a^{-3}$, so the annihilation rate drops more rapidly than the expansion rate as the scale factor $a$ increases. Thus unless annihilations are effective immediately after the end of inflation, they will never be significant and inflaton decays will be the main mode of reheating. However, if the inflaton condensate fragments soon after oscillations begin then the value of the amplitude $s$ inside the lumps is constant and so $\Gamma_{a n n} / H \propto a^{3 / 2}$ increases as $a$ increases. Therefore as the Universe expands annihilations will eventually occur and may be the dominant mode of reheating. The reheating temperature due to annihilations is then

$$
T_{R}=\left(\frac{45}{4 \pi^{3} g\left(T_{d}\right)}\right)^{1 / 4}\left(\Gamma_{d} M_{P l}\right)^{1 / 2}=\left(\frac{1}{128 \pi}\right)^{1 / 2}\left(\frac{M_{P l}}{m_{S}}\right)^{1 / 2}\left(\frac{45}{4 \pi^{3} g\left(T_{d}\right)}\right)^{1 / 4} \lambda^{2} s_{c},
$$

where we have used $s \approx s_{c}$ inside the lumps and where $g\left(T_{d}\right)$ is the number of degrees of freedom in thermal equilibrium [5].

We next apply this to the specific case of D-term inflation. If the MSSM fields also carry $U(1)_{F I}$ charges, there is an interaction with the scalars of the MSSM of the form $\lambda^{2}\left|Q_{i}\right|^{2}|S|^{2}$ coming from integrating out the $U(1)_{F I}$ gauge fields [16], where $\lambda$ is the superpotential coupling from Eq. (11). The reheating temperature is then

$$
T_{R}=\left(\frac{1}{64 \pi}\right)^{1 / 2}\left(\frac{45}{4 \pi^{3} g\left(T_{d}\right)}\right)^{1 / 4} g \lambda^{1 / 2} \xi^{1 / 4} M_{P l}^{1 / 2} \approx 5.5 \times 10^{15}\left(\frac{100}{g\left(T_{R}\right)}\right)^{1 / 4} g \lambda^{1 / 2} \mathrm{GeV} .
$$

The upper bound from requirng that gravitinos are not generated excessively by thermal scattering is $T_{R} \lesssim 10^{8-9} \mathrm{GeV}$ [27]. Thus we require

$$
g \lambda^{1 / 2} \lesssim 1.8 \times 10^{-7}\left(\frac{g\left(T_{R}\right)}{100}\right)^{1 / 4}\left(\frac{T_{R}}{10^{9} \mathrm{GeV}}\right) .
$$


The smallest value of $\lambda$ for which fragmentation occurs is $\lambda \approx 0.2 g$. In this case if condensate fragmentation occurs then from Eq. (49) the $U(1)_{F I}$ gauge coupling must satisfy $g \lesssim 7 \times 10^{-5}$. (This upper bound will be even stronger in the presence of parametric resonance.) This will not be satisfied if, for example, the $U(1)_{F I}$ gauge coupling has the typical magnitude $g \approx 1$ of the MSSM gauge couplings. Thus condensate fragmentation is typically not compatible with D-term inflation if the inflatons can annihilate to MSSM fields. In order to have D-term inflation consistent with the absence of thermal gravitinos we must either eliminate inflaton annihilations, which requires that the MSSM fields do not carry $U(1)_{F I}$ charges, or eliminate inflaton condensate fragmentation, which requires that $\lambda<0.2 g$.

\section{Inflaton Condensate Fragmentation and Tachy- onic Preheating}

Throughout the preceeding discussion we have assumed that a homogeneous inflaton condensate forms at the end of inflation. However, it has been shown that spatial perturbations of the inflaton field may grow and become non-linear much more rapidly (before homogeneous oscillations are established) in a process known as tachyonic preheating [17, 18]. In this case the final state is composed of colliding scalar field waves [17, 18]. Recently it has also been shown [19] that condensate lumps occur in tachyonic preheating (called "oscillating hot spots" in [19]). Tachyonic preheating typically occurs in less than the time for a single coherent oscillation [17], making it impossible to average over a coherently oscillating inflaton field. Inflaton condensate fragmentation and tachyonic preheating may be regarded as different manifestations of a general phenomena, namely the instability of the inflaton field with respect to spatial perturbations in hybrid inflation models.

The question of whether inflaton condensate fragmentation or tachyonic preheating occurs at the end of hybrid inflation will depend upon the state of the field at the time when the inflaton reaches $s_{c}$, in particular the rate at which the homogeneous field $s(t)$ is rolling (where $s=s(t)+\delta s(\mathbf{x}, t)$ ) relative to the rate of tachyonic growth of the 
spatial perturbations $\delta s(\mathbf{x}, t)$ at $s<s_{c}$. If the homogeneous field can catch up with the spatial perturbations (which cross $s_{c}$ before the homogeneous field) before there is significant growth of the perturbations due to the tachyonic mass term at $s<s_{c}$, then there will be no tachyonic preheating. This depends crucially on the rate of rolling of the homogeneous field at $s_{c}$, which in turn requires that the full inflaton potential with radiative corrections be considered. For the case of D-term inflation a full analysis has yet to be done [20].

\section{Conclusions}

We have shown that it is possible for the inflaton condensate in hybrid inflation models to fragment to condensate lumps. The inflaton condensate is in general unstable, but the instability reduces as the Universe expands, requiring that fragmentation occurs shortly after the end of inflation. The state of the Universe after fragmentation, with the energy density concentrated inside inflaton condensate lumps, is quite different from the conventional post-inflation scenario of a homogeneous inflaton condensate. One consequence of inflaton condensate fragmentation is that inflaton annihilations will be effectively enhanced relative to the case of a homogeneous inflaton condensate. In the case of D-term inflation models, which we have used as a specific example of hybrid inflation models in our discussion, if condensate formation and fragmentation occurs then the enhancement of inflaton annihilations implies that the reheating temperature is typically large compared with the thermal gravitino upper bound. Thus either inflaton annihilations must be suppressed, which requires that the MSSM fields do not carry $U(1)_{F I}$ gauge charges, or fragmentation must not occur, which requires that $\lambda<0.2 g$.

If inflaton annihilations do not occur, or if we consider a non-SUSY model for which there is no thermal gravitino bound on the reheating temperature, then inflaton condensate fragmentation can safely occur. This may have interesting consequences for the inflaton dominated period following inflation. For example, the fact that the energy density of the Universe is now concentrated in condensate lumps could greatly alter the 
dynamics of SUSY flat direction scalar fields, which acquire a mass from the SUSY breaking inflaton energy density in the case of a homogeneous inflaton condensate [3]. Another potentially important effect would be for parametric resonant decay of the inflaton and preheating. In the case of a homogeneous condensate, parametric resonance turns off as the Universe expands and the inflaton oscillation amplitude decreases [28, 29]. However, if the condensate fragments then, just as in our discussion of perturbative annihilations, the oscillation amplitude will be frozen inside the lumps and so parametric resonant decay should continue without stopping, resulting in more efficient preheating.

Growth of spatial perturbations of the inflaton field in hybrid inflation models has also been demonstrated in the context of tachyonic preheating. In general, the mode by which hybrid inflation ends is likely to be sensitive to both the model paramaters and the initial conditions at the end of inflation. A detailed numerical investigation will therefore be necessary in order to clarify how hybrid inflation ends in a given model [20]. However, it should be emphasized that condensate lumps are a feature of both inflaton condensate fragmentation and tachyonic preheating [19]. Therefore our discussion of the cosmology of condensate lumps should apply to the tachyonic preheating case also.

It is important to emphasize that inflaton condensate fragmentation is a natural possibility in all hybrid inflation models and that it may have consequences for cosmology beyond what has been discussed here. A detailed understanding of inflaton condensate fragmentation will therefore be necessary in order to fully understand the cosmology of hybrid inflation models.

\section{References}

[1] M.S.Turner, Phys. Rev. D33 (1986) 889; J.E.Kim, Phys. Rep. 150 (1987) 1.

[2] I.A.Affleck and M.Dine, Nucl. Phys. B249 (1985) 361.

[3] M.Dine, L.Randall and S.Thomas, Nucl. Phys. B458 (1996) 291. 
[4] T.Gherghetta, C.Kolda and S.P.Martin, Nucl. Phys. B468 (1996) 37.

[5] E.W. Kolb and M.S. Turner, The Early Universe (Addison-Wesley, Reading MA, USA, 1990).

[6] E.Kolb and I.Tkachev, Phys. Rev. Lett. 71 (1993) 3051; Phys. Rev. D49 (1994) 5040.

[7] G.Dvali, A.Kusenko and M.Shaposhnikov, Phys. Lett. B417 (1998) 99.

[8] A.Kusenko and M.Shaposhnikov, Phys. Lett. B418 (1998) 46.

[9] K.Enqvist and J.McDonald, Phys. Lett. B425 (1998) 309.

[10] K.Enqvist and J.McDonald, Nucl. Phys. B538 (1999) 321.

[11] S.Kasuya and M.Kawasaki, Phys. Rev. D61 (2000) 041301; Phys. Rev. D62 (2000) 023512

[12] A.Linde, Phys. Rev. D49 (1994) 748.

[13] E.J.Copeland, A.R.Liddle, D.H.Lyth, E.D.Stewart and D.Wands, Phys. Rev. D49 (1994) 6410; G.Dvali, Q.Shafi and R.Schaefer, Phys. Rev. Lett. 73 (1994) 1886.

[14] E.Halyo, Phys. Lett. B387 (1996) 43; P.Binetruy and G.Dvali, Phys. Lett. B388 (1996) 241.

[15] D.Lyth and A.Riotto, Phys. Rep. 314 (1999) 1.

[16] C.Kolda and J.March-Russell, Phys. Rev. D60 (1999) 023504.

[17] G.Felder, J.Garcia-Bellido, P.B.Greene, L.Kofman, A.Linde and I.Tkachev, Phys. Rev. Lett. 87 (2001) 011601; G.Felder and L.Kofman, Phys. Rev. D63 (2001) 103503; G.Felder, L.Kofman and A.Linde, hep-th/0106179.

[18] L.Kofman, hep-ph/0107280.

[19] E.J.Copeland, S.Pascoli and A.Rajantie, hep-ph/0202031. 
[20] M.Broadhead and J.McDonald, In Progress.

[21] M.Turner, Phys. Rev. D D28 (1983) 1243.

[22] J.McDonald, Phys. Rev. D48 (1993) 2573.

[23] D.Lyth, Phys. Lett. B469 (1999) 69.

[24] M.Bastero-Gil, S.King and J.Sanderson, Phys. Rev. D60 (1999) 103517.

[25] K.Lee, Phys. Rev. D50 (1994) 5333.

[26] J.McDonald, Phys. Rev. D50 (1994) 3637.

[27] J.Ellis, J.E.Kim and D.V.Nanopoulos, Phys. Lett. B145 (1984) 181; S.Sarkar, Rep. Prog. Phys. 59 (1996) 1493.

[28] S.Y.Khlebnikov and I.I.Tkachev, Phys. Rev. D56 (1997) 653; L.Kofman, A.D.Linde and A.A.Starobinsky, Phys. Rev. D56 (1997) 3258.

[29] I.Zlatev, G.Huey and P.J.Steinhardt, Phys. Rev. D57 (1998) 2152. 